\title{
Hacia una evaluación docente universitaria por competencias
}

Towards a University Teacher Evaluation by Competencies

Rumo a uma avaliação do professor universitário por competências

\author{
Alma Lilia Sapién Aguilar \\ Universidad Autónoma de Chihuahua, México \\ Isapien@uach.mx \\ https://orcid.org/0000-0001-7222-2612 \\ Laura Cristina Piñón Howlet \\ Universidad Autónoma de Chihuahua, México \\ Ipinon@uach.mx \\ https://orcid.org/0000-0002-1176-2567 \\ Silvia Patricia Aguirre Espino \\ Universidad Autónoma de Chihuahua, México \\ saguirr@uach.mx \\ https://orcid.org/0000-0003-2860-874X \\ Luis Antonio Molina Corral \\ Universidad Autónoma de Chihuahua, México \\ Imolin@uach.mx \\ https://orcid.org/0000-0002-3217-6460
}




\section{Resumen}

La evaluación de competencias docentes es un proceso necesario para mejorar la calidad educativa de nivel superior. El objetivo de la presente investigación fue analizar las competencias docentes de la plantilla de profesores de la Facultad de Contaduría y Administración (FCA) de la Universidad Autónoma de Chihuahua (UACH). La investigación fue de naturaleza cuantitativa; el diseño fue no experimental, transeccional descriptivo, y el muestreo fue probabilístico. Para ello, además, se realizaron consultas bibliográficas e investigación de campo. Así, con una muestra de 230 docentes, se describió y analizó la situación actual de las competencias docentes en la FCA. Los principales resultados señalan la necesidad de implementar programas de capacitación adecuados a los docentes, ya que se cuenta con áreas de oportunidad que se deben fortalecer, así como tomar en cuenta la influencia que ejercen la edad y la situación laboral de los docentes.

Palabras clave: competencia docente, evaluación docente, universidad.

\section{Abstract}

The evaluation of teaching competencies is a necessary process to improve the quality of higher education. The objective of this research was to analyze the teaching skills of the teaching staff of the Faculty of Accounting and Administration (FCA) of the Universidad Autónoma de Chihuahua. The research was quantitative in nature; the design was nonexperimental, cross-sectional descriptive, and the sampling was probabilistic. For this, in addition, bibliographic consultations and field research were carried out. Thus, with a sample of 230 teachers, the current situation of teaching competencies in the FCA was described and analyzed. The main results indicate the need to implement appropriate training programs for teachers, since there are areas of opportunity that must be strengthened, as well as taking into account the influence of teachers' age and employment status.

Keywords: teacher competence, teacher evaluation, university. 


\section{Resumo}

A avaliação das competências docentes é um processo necessário para melhorar a qualidade do ensino superior. $\mathrm{O}$ objetivo desta pesquisa foi analisar a capacidade docente do corpo docente da Faculdade de Contabilidade e Administração (FCA) da Universidade Autônoma de Chihuahua (UACH). A pesquisa foi de natureza quantitativa; o delineamento foi não experimental, transversal descritivo e a amostragem foi probabilística. Para tanto, foram realizadas consultas bibliográficas e pesquisas de campo. Assim, com uma amostra de 230 professores, foi descrita e analisada a situação atual das competências docentes na FCA. Os principais resultados apontam para a necessidade de implementação de programas adequados de formação de professores, uma vez que existem áreas de oportunidades que devem ser fortalecidas, bem como levando em consideração a influência da idade dos professores e da situação de emprego.

Palavras-chave: competência docente, avaliação de professores, universidade.

Fecha Recepción: Enero 2021

Fecha Aceptación: Septiembre 2021

\section{Introducción}

La actividad del docente implica el compromiso de contar con las competencias necesarias, suficientes y pertinentes para llevar a cabo con calidad la educación en México, así como para afrontar los retos y oportunidades que esta presenta. Los docentes tienen la responsabilidad social de contribuir con la formación de los estudiantes, una de tipo integral y humanística, por medio de competencias que vayan más allá de lo profesional. Para ello el proceso enseñanza-aprendizaje debe tener sentido tanto para alumnos como para los propios docentes.

Actualmente, la institución educativa ya no es el único canal mediante el cual las nuevas generaciones entran en contacto con el conocimiento y la información (García y Vaillant, 2010). Por lo anterior, los docentes requieren de nuevos escenarios formativos. Las prácticas docentes se deben actualizar tanto en el contenido como en la forma. La figura del profesor ahora debe ser la de un intermediario entre los contenidos y los alumnos, tomando en cuenta la diversidad de situaciones en las cuales se encuentran inmersos. Ahora quizá más que nunca el desarrollo de los docentes en servicio y la formación de la nueva generación de académicos constituyen cuestiones de suma importancia (Brunner, 1994). El método educativo, en Latinoamérica y en todo el mundo, se encuentra enérgicamente impactado por el fenómeno de la globalización y la nueva sociedad del conocimiento. Los modelos educativos y las tecnologías utilizadas están creciendo en cuanto a pluralidad y capacidad (Inche y Chung, 2012). 


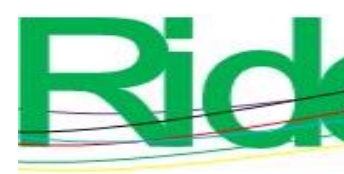

Revista Iberoamericana para la Investigación y el Desarrollo Educativo ISSN $2007-7467$

Hoy en día, la educación debe enfocarse aún más en que cada individuo desarrolle activamente sus propias competencias, lo que involucra un estímulo a la imaginación y creatividad, así como al pensamiento crítico y la resolución de problemas que demandan el contexto actual y también futuro (Carrera y Marín, 2011). Para consumar el desarrollo del alumno y mejorar su calidad educativa, se necesita por parte del docente experiencia, conocimiento e interacción entre los involucrados, entre este y aquel.

En el presente los sistemas de educación superior están siendo enfrentados a fuertes mandatos para elevar la calidad de su enseñanza al grado que ésta se ha convertido en su prioridad estratégica. La discusión acerca de los modelos educativos basados en normas de competencia y su desempeño para mejorar la relación entre la educación y el funcionamiento están centrándose en la necesidad de impulsar un mayor nivel de flexibilidad en los sistemas educativos. Consecuentemente, en las estrategias de diferentes países puede observarse la integración de diversos subsistemas interactivos; de gran interés son los planes educativos modulares y los procesos de acreditación para utilizar con flexibilidad la oferta educativa en tiempos y espacios desiguales (Rojas, 2000).

Hay que estar preparado en cuanto a los procesos de enseñanza-aprendizaje para desarrollarse como un docente universitario. La acreditación de la institución y de sus diferentes carreras es un aspecto de mucha importancia, aunque lo es igualmente la supervisión de la docencia y cómo esta se imparte, además de evaluar los métodos pedagógicos y las estrategias pedagógicas. Así, se asegura la calidad de los aprendizajes en los diferentes espacios educativos (Montenegro y Fuentealba, 2010). Los procesos de evaluación responden a propósitos determinados que llevan a un fin de acuerdo con la naturaleza de sus objetivos. Se trata de una acción predominantemente humana donde se presenta un grado de subjetividad con los sujetos implicados en dicho proceso (Fernández, Díaz y Leyva, 2016). Además, el docente debe valorar sus saberes y elegir los aspectos destacados, metodologías y el mejor momento para llevar a cabo la evaluación (Mas y Olmos, 2016). Es necesaria, además de la evaluación externa, encaminada al desarrollo profesional, una autoevaluación del docente (Marchesi y Pérez, 2018).

Tras las bambalinas institucionales, puede apreciarse que son demandadas las capacitaciones docentes de forma permanente, pero es vital saber en qué rubros del proceso de enseñanza-aprendizaje deben ser aplicadas. Para lo cual, es vital que se conozcan las competencias docentes objetivo, así como tener un parámetro de su estado actual para estar al tanto de cuáles requieren mayor trabajo de formación. Debe también 
considerarse una retroalimentación para el docente universitario, según Mas (2011), cuyo flujo se presenta a continuación en la figura 1.

Figura 1. Retroalimentación del docente universitario

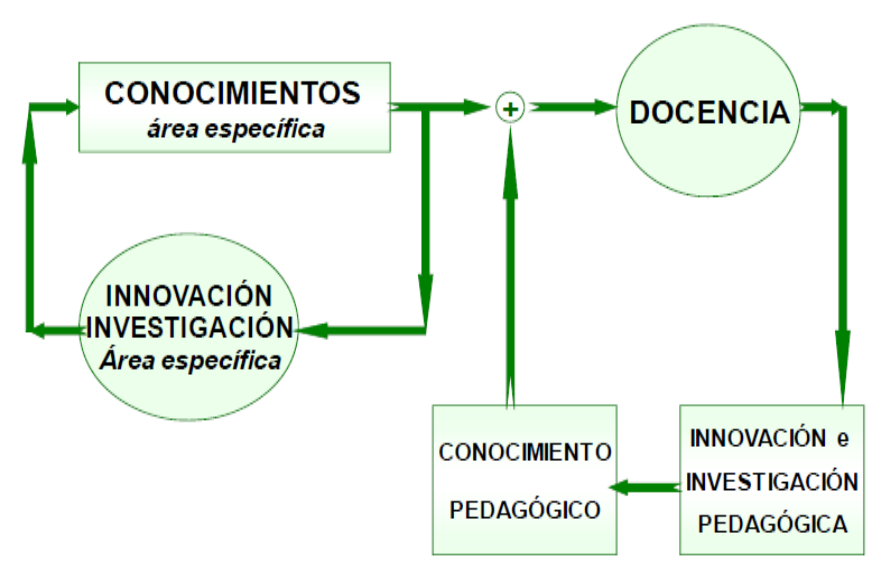

Fuente: Tomado de "El profesor universitario: sus competencias y formación” (p. 11), por Ó. Mas, 2011, Profesorado. Revista de Currículum y Formación de Profesorado, $15(3)$.

Ahora bien, a partir de la década de los 90, los docentes de la Facultad de Contaduría y Administración (FCA) de la Universidad Autónoma de Chihuahua (UACH) comenzaron a guiarse con el modelo educativo por competencias centrado en el aprendizaje. Cabe señalar que la organización de dicho enfoque está asentada en cuatro diferentes pilares: filosófico, conceptual, psicopedagógico y metodológico (Marín, 2003). Este modelo educativo, presentado en la figura 2, considera que todo ser humano tiene una gran serie de capacidades susceptibles a ser desarrolladas cuando muestra interés por aprender.

Figura 2. Modelo educativo

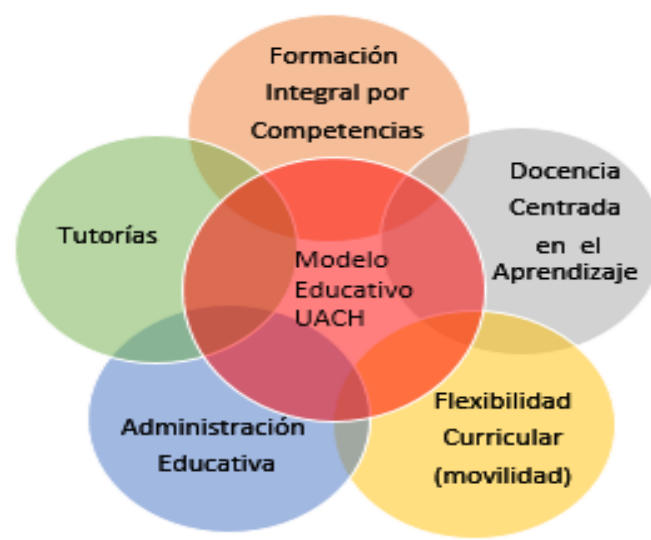

Fuente: Tomado de El modelo educativo de la UACH: elementos para su construcción, por R. Marín, 2003, México: UACH/Dirección académica. 


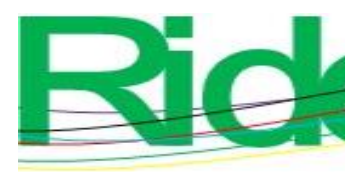

Revista Iberoamericana para la Investigación y el Desarrollo Educativo ISSN 2007 - 7467

Conjuntamente, coloca el diseño y rediseño curricular por competencias desde una perspectiva abierta y flexible, aunado al acompañamiento del alumno por medio de tutorías y una gestión educativa enfocada al cambio. El enfoque teórico manejado en las competencias docentes incluye los tres saberes de los que habla Tobón (2004): saber ser, saber conocer y saber hacer.

En relación con la constante exigencia hacia las universidades sobre proporcionar una educación de calidad, la evaluación de competencias docentes es una parte esencial para poder mejorar este requerimiento. Sobre todo, se debe entender y estar consciente de cuál es el papel del docente y cómo se encuentra su desempeño. Por lo anterior, surge el planteamiento de las siguientes preguntas de investigación: 1) ¿cuál es el análisis de la evaluación docente universitaria por competencias?; 2) ¿es posible destacar las necesidades de capacitación en los docentes?, y 3) ¿existe una influencia de la edad y situación laboral en el desempeño docente?

El objetivo general de esta investigación fue analizar la evaluación de competencias docentes en la FCA de la UACH. Los objetivos específicos fueron: 1) analizar la evaluación docente universitaria por competencias; 2) destacar las necesidades de capacitación en los docentes en las diferentes dimensiones en que se dividen las competencias, y 3) encontrar si existe una influencia de la edad y la situación laboral en el desempeño docente.

\section{Marco teórico}

Hace algunas décadas bastaba con que los académicos que educaban en las universidades fueran expertos en sus materias; el aprendizaje de los estudiantes se consideraba como un tema menor (Ashwin, 2006). No obstante, hoy se espera que los académicos y otros involucrados en la formación profesional estén preparados para desempeñar su papel como educadores tanto en los procesos de enseñanza-aprendizaje como en los contenidos que instruyen.

La docencia, como ya se había mencionado anteriormente, es uno de los criterios de evaluación para la acreditación de carreras profesionales y sus programas. Como parte de ello, se evalúan los métodos pedagógicos y las estrategias de enseñanza-aprendizaje, al igual que los resultados obtenidos en el uso de dichas metodologías, con la intención de afirmar o corroborar la calidad de los aprendizajes en estos espacios educativos (Comisión Nacional de Acreditación de Chile, 2016). Igualmente, las competencias ligadas con la gestión de la enseñanza y sus recursos, al mismo tiempo que las sociales, aquellas que benefician acciones de cooperación, liderazgo y trabajo en equipo. Por 


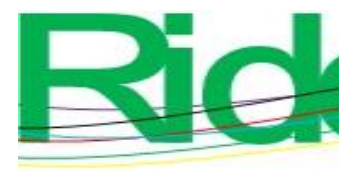

Revista Iberoamericana para la Investigación y el Desarrollo Educativo ISSN 2007 - 7467

último, las competencias afectivas que garantizan actitudes, conductas y motivaciones que sustentan la responsabilidad y el compromiso de la docencia con los objetivos de formación (Toro, Saldarriaga, León, Martínez y Arias, 2015).

En la formación de competencias, la responsabilidad incluye a las instituciones educativas y también a la sociedad, el sector laboral empresarial, la familia y la persona involucrada, esto es, el estudiante (Tobón, 2004). En las instituciones, en la práctica, uno de los criterios principales continúa siendo la vinculación y especialización de docentes con la disciplina a impartir, dejando de lado la formación pedagógica. Esta dinámica conlleva a que la docencia universitaria se centre en la enseñanza de las disciplinas, no dando importancia adecuada a los procesos como la formación por competencias y el uso de tecnologías de la información y la comunicación (TIC). Evidentemente, no es factible exigir que todas estas competencias se den en una persona, por lo que será necesaria la disposición de equipos de trabajo en los que pueda darse la especialización en algunas de ellas.

Sea cual fuere el modelo educativo que se aplique en una institución universitaria, la planificación es un elemento importante como función básica del proceso de enseñanza-aprendizaje. Las competencias tecnológicas son, asimismo, un factor de cambio social ya que contribuyen a la expansión y diversificación de la educación. El modelo por competencias ha dejado al descubierto una serie de requisitos que antes no se utilizaban o no eran tomados en cuenta: el saber, el saber hacer, el saber estar y el saber ser. El docente se convierte en un actor principal y transformador de ese proceso a través del cual, se pretende, los estudiantes pasen a ser sujetos integrales, con la capacidad de desempeñarse en un mundo que cambia a una velocidad antes no vista, rápida y constantemente, además de poseer los conocimientos, las habilidades y la destreza para poder ser competitivo en un entorno globalizado (Durán, 2016).

Un referente que vale la pena tener en mente es el modelo holístico de competencia docente para el mundo digital de Esteve, Castañeda y Adell (2018), que se conforma de seis componentes: 1) experto en contenidos pedagógicos digitales, 2) generador y gestor de prácticas pedagógicas emergentes, 3) capaz de usar las TIC para expandir su relación con la familia y el entorno con el estudiante, 4) práctico reflexivo aumentado, 5) experto en entornos de aprendizaje enriquecidos y 6) sensible al uso de la tecnología desde la perspectiva del compromiso social. Un solo modelo no puede detallar de forma cierta todos los posibles quehaceres docentes en todos los niveles educativos, por tanto, el modelo desarrolla la función docente dentro de la enseñanza formal en un 


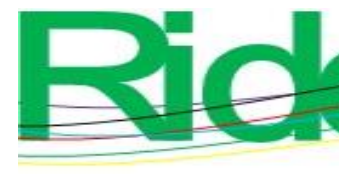

Revista Iberoamericana para la Investigación y el Desarrollo Educativo ISSN $2007-7467$

nivel preuniversitario. En este modelo, el docente es un profesional comprometido con su profesión y con el entorno en el que se desenvuelve.

Los docentes de nivel superior deben contar con habilidades, conocimientos, actitudes y valores necesarios para realizar una instrucción de calidad. Además, deben emprender de forma satisfactoria los problemas que la enseñanza implica como lo es reflexionar y construir los conocimientos con la aplicación de los tres saberes ya mencionados de Tobón (2004). El saber profesional se puede interpretar como un modelo que explique los conocimientos o teorías profesionales que guían la acción; de igual forma, está orientado por teorías científicas y subjetivas que se encuentran poco desarrolladas en el proceso formativo y de investigación (Cuadra, Castro y Juliá, 2018).

La evaluación de la docencia en México es considerada un proceso que se lleva a cabo por instituciones de nivel superior con fines administrativos. De forma externa se recurre a organismos evaluadores, los cuales otorgan acreditaciones, y programas de formación de profesores. Internamente, proporciona información para la toma de decisiones y estímulos económicos a los docentes. En cuestiones académicas, la información sirve para brindar capacitación y actualización a los docentes, sí, sin embargo, los resultados y necesidades de mejorar habilidades pedagógicas en cuanto a planeación, conducción y evaluación del aprendizaje no están vinculados con la formación para una educación de calidad (Marín, Guzmán, Márquez y Peña, 2013).

La progresión del modelo por competencias en la educación superior tiene como intención impulsar el desarrollo de estas en los egresados universitarios, según Dios, Calmaestra y Rodríguez (2018). La identificación de competencias en los docentes permite edificar el perfil de competencias. Estas se consideran aquí tal cual como son descritas en el modelo para el desarrollo y evaluación de competencias académicas (DECA) validado por Guzmán, Marín e Inciarte (2014). El saber docente es plural y se despliega hacia diferentes dimensiones, que se retroalimentan y actualizan. Las preguntas que deberían orientar el trabajo docente hoy en día son qué capacidades demanda el siglo XXI y qué ciudadanos queremos y necesitamos (Fondo, 2019). 


\section{Metodología}

La naturaleza o enfoque de la investigación fue cuantitativa de tipo aplicada. Asimismo, fue transeccional o transversal. El trabajo de investigación se realizó en la FCA de la UACH. El tipo de muestreo fue probabilístico. El diseño fue no experimental, de campo, apoyado en la consulta de fuentes electrónicas. Para la validación de la estrategia, se usó la recolección de datos mediante la aplicación del instrumento, que contiene datos generales y ocho competencias docentes divididas en cuatro dimensiones; en total, 44 preguntas que fueron cerradas para medirse con una escala de Likert construida a partir de los siguientes valores: $0=$ Nada, $1=$ Poco, $2=$ Regular, $3=$ Bastante, 4 = Excelente; y de opción múltiple para los datos generales. La recolección de la información se llevó a cabo en Excel y la codificación para los estadísticos se realizó por medio del software SPSS versión 22. El instrumento arrojó un alfa de Cronbach de aceptación de 0.930. El proceso de validación de los ítems, por su parte, fue a partir de índices de univocidad y pertinencia, cuyos resultados se posicionaron en niveles desde 0.90 hasta 1.00, donde el rango del valor máximo es uno y el mínimo cero (Carrera, Vaquero y Balsells, 2011). El instrumento utilizado se tomó de una encuesta elaborada por Valles et al. (2017) durante una investigación realizada sobre competencias docentes en la UACH.

Se ejecutó un muestreo por estratificación, dividiendo la población en estratos a partir de los indicadores de edad y situación laboral obtenidos en los datos generales del instrumento aplicado. Luego se llevó a cabo un análisis de clúster o de conglomerados jerárquicos para establecer grupos en función a un conjunto de variables que, en este caso, fueron la evaluación del desempeño de las competencias docentes por dimensiones desarrolladas en la FCA de la UACH con respecto a los grupos de edad y situación laboral. Se determinó el número de grupos, su descripción y validación, además del gráfico de clúster por dendrograma. Después, se realizó el análisis de k-medias y, por último, se hizo un análisis de brecha que sirvió para corroborar el resultado a partir de los datos recolectados y comparar el desempeño desarrollado con el desempeño esperado o ideal, todo lo cual contribuyó al diseño de una estrategia. La variable independiente analizada fue la evaluación de competencias docentes (causa), la cual no fue manipulada. Mientras que las variables dependientes (efecto) fueron las ocho distintas competencias que se dividieron en las siguientes dimensiones: Docencia, Investigación, Tutoría y Gestión (figura 3). La muestra fue de 230 participantes y fue calculada con un nivel de confianza de $95 \%$. 
Figura 3. Variables

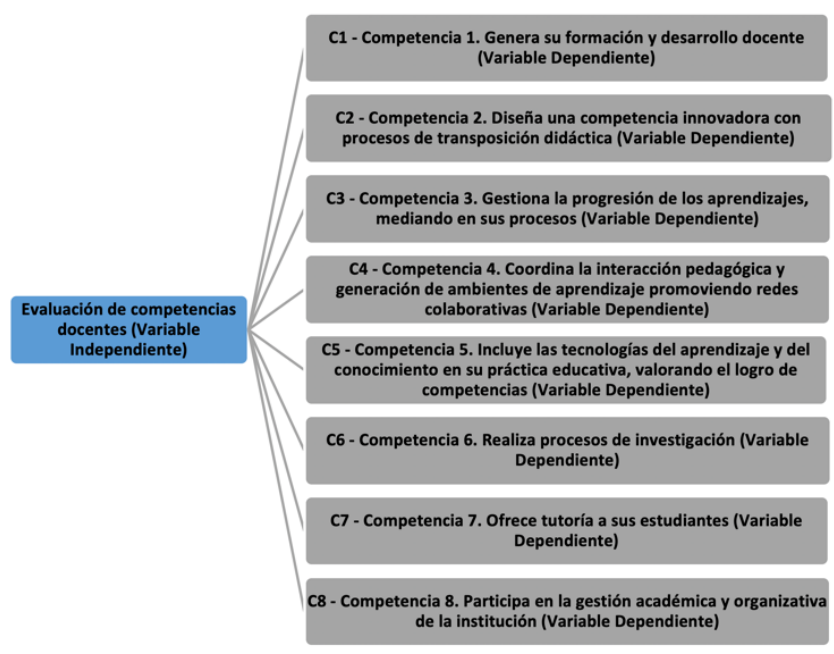

Fuente: Elaboración propia

\section{Resultados}

Primordialmente, para poder evaluar las competencias desarrolladas por los docentes de la FCA de la UACH, se parte de que dichas competencias están divididas en cuatro dimensiones, a saber: Docencia, Investigación, Tutoría y Gestión.

La fiabilidad de consistencia interna del instrumento se estimó mediante el alfa de Cronbach, que reportó resultados aceptables $(\alpha \geq 0.70)$, tomando como criterio las recomendaciones de George y Mallery (2003, citados en Hernández y Pascual, 2018). El instrumento demostró ser consistente en la validez y confiabilidad como se muestra en las tablas 1 y 2 .

Tabla 1. Escala: confiabilidad

\begin{tabular}{|c|c|c|c|}
\hline & & $\mathrm{N}$ & $\%$ \\
\hline Casos & Válido & 227 & 98.7 \\
\hline & Excluidos ${ }^{\mathrm{a}}$ & 3 & 1.3 \\
\hline & Total & 230 & 100.0 \\
\hline $\begin{array}{l}{ }^{\mathrm{a}} \text { La eli } \\
\text { La ex } \\
\text { estuvie } \\
\text { instrun }\end{array}$ & $\begin{array}{l}\text { inación por } \\
\text { sión de t } \\
\text { n fuera de } \\
\text { to. }\end{array}$ & $\begin{array}{l}\text { basa } \\
\text { cipa } \\
\text { res }\end{array}$ & $\begin{array}{l}\text { das las variables del procedimiento. } \\
\text { fue debido a que las respuestas } \\
\text { escala de Likert propuestos para el }\end{array}$ \\
\hline
\end{tabular}

Fuente: Elaboración propia 
Tabla 2. Estadísticas de fiabilidad

\begin{tabular}{|l|l|l|}
\hline Alfa de Cronbach & $\begin{array}{l}\text { Alfa de Cronbach } \\
\text { basada en elementos } \\
\text { estandarizados }\end{array}$ & Número de elementos \\
\hline 0.930 & 0.934 & 44 \\
\hline
\end{tabular}

Fuente: Elaboración propia

El muestreo aleatorio por estratificación se presenta en las tablas 3 y 4, donde se muestra la frecuencia de los indicadores de edad y situación laboral.

Tabla 3. Edad

\begin{tabular}{|l|l|l|l|}
\hline \multicolumn{2}{|c|}{} & Frecuencia & $\begin{array}{l}\text { Frecuencia } \\
\text { acumulada }\end{array}$ \\
\hline Válido & De 20 a 29 años & 26 & 26 \\
\cline { 2 - 4 } & De 30 a 39 años & 54 & 80 \\
\cline { 2 - 4 } & De 40 a 49 años & 60 & 140 \\
\cline { 2 - 4 } & De 50 a 59 años & 57 & 57 \\
\cline { 2 - 4 } & De 60 a 69 años & 30 & 87 \\
\cline { 2 - 4 } & Total & 227 & 227 \\
\hline Perdidos & Sistema & 3 & 1.3 \\
\hline Total & 230 & 100.0 \\
\hline
\end{tabular}

Fuente: Elaboración propia

El siguiente paso es multiplicar la frecuencia acumulada de 40 a 49 años por el coeficiente, que es igual al tamaño de la muestra entre la población, 230/543 $=0.42 \times 140$ $=59$, esto es, el tamaño de la muestra aleatoria por estratificación. Mientras que, para la frecuencia acumulada de 60 a 69 años, la muestra aleatoria por estratificación es de 37. Y al fusionarlas, queda una muestra aleatoria por estratificación de 96. 
Tabla 4. Situación laboral

\begin{tabular}{|l|l|l|l|}
\hline \multicolumn{2}{|c|}{} & Frecuencia & $\begin{array}{l}\text { Frecuencia } \\
\text { acumulada }\end{array}$ \\
\hline Válido & $\begin{array}{l}\text { Profesor de tiempo } \\
\text { completo }\end{array}$ & 33 & 33 \\
\cline { 2 - 4 } & Profesor de hora clase & 129 & 129 \\
\cline { 2 - 4 } & Administrativo & 26 & 155 \\
\cline { 2 - 4 } & Contratado & 36 & 191 \\
\cline { 2 - 4 } & Técnico & 1 & 192 \\
\cline { 2 - 4 } & Profesor de medio tiempo & 2 & 194 \\
\cline { 2 - 4 } & Total & 227 & 227 \\
\hline Perdidos & Sistema & 3 & 1.3 \\
\hline Total & & 230 & 100.0 \\
\hline
\end{tabular}

Fuente: Elaboración propia

A continuación, se multiplica la frecuencia acumulada de 33 profesores de tiempo completo por el coeficiente, que es igual al tamaño de la muestra entre la población, $230 / 543=0.42 \times 33=14$, esto es, el tamaño de la muestra aleatoria por estratificación. Mientras que para la frecuencia acumulada de profesores hora clase, administrativo, contrato, técnico y profesor de medio tiempo, la muestra aleatoria por estratificación es de 83. Y al fusionarlas, queda una muestra aleatoria por estratificación de 97.

Para el análisis de clúster o conglomerados jerárquicos, se obtuvieron tres grupos conformados de manera homogénea por medio de un dendrograma que utiliza la vinculación de Ward; en las características del clúster por dimensiones, las puntuaciones más altas son tres y cuatro, por lo que tienen inclinación positiva hacia la pregunta y puntuaciones de uno a dos. Así, el conglomerado uno pertenece a docentes que solamente tienen una inclinación positiva en cinco de los indicadores de la dimensión Docencia, mientras que en las otras dimensiones su inclinación es baja. En el conglomerado dos, son docentes con una inclinación positiva en todos los indicadores de todas las dimensiones, y por último, el conglomerado tres tiene una inclinación positiva en casi todos los indicadores de la dimensión Docencia; solamente se hace una excepción en dos indicadores, para la dimensión Investigación solamente tiene inclinación positiva en dos indicadores e inclinación baja para las dimensiones de Tutorías y Gestión. Las características del grupo tres fueron altas en puntuación de las variables en cuanto al desempeño de las competencias docentes de la dimensión Docencia en seis de los 17 indicadores. El grupo dos tuvo calificaciones altas en las competencias de todas las 
dimensiones. Y, por último, el grupo tres, cuenta con 15 de los 17 indicadores con evaluaciones en nivel alto de las competencias de la dimensión Docencia. Cabe destacar que el grupo dos estuvo conformado por 23 sujetos de estudio, de los cuales 15 pertenecen a una edad de 50 años o más y ocho pertenecen a la edad de 49 años o menos. De la muestra total de 230, $62 \%$ pertenece al segundo grupo, mientras que $38 \%$ son sujetos de 50 o más años de edad. Por lo que la edad influye en la evaluación del desempeño docente de todas las dimensiones. Con respecto a la influencia que pueda tener la situación laboral con el desempeño docente, se llevó a cabo el cálculo de k-medias y dio como resultado, de acuerdo con el análisis de varianza (Anova), que la variable ha aportado significativamente a la discriminación en el clúster, así como que las distancias son separadas en los centros (Tabla 5). Y, por último, en el conglomerado dos se encuentran los docentes que tienen una inclinación más positiva en todos los indicadores de todas las dimensiones. Por lo que la forma de contratación o situación laboral también influye en el desempeño de los docentes.

Tabla 5. Distancias entre centros de clústeres finales

\begin{tabular}{|l|l|l|l|}
\hline Clúster & 1 & 2 & 3 \\
\hline 1 & & 8.142 & 5.663 \\
\hline 2 & 8.142 & & 7.111 \\
\hline 3 & 5.663 & 7.111 & \\
\hline
\end{tabular}

Fuente: Elaboración propia

Los conglomerados tienen diferencias entre la situación laboral de docente de tiempo completo y las demás formas de contratación.

Tabla 6. Número de casos en cada clúster

\begin{tabular}{|l|l|l|}
\hline Clúster & 1 & 15.000 \\
\cline { 2 - 3 } & 2 & 51.000 \\
\cline { 2 - 3 } & 3 & 31.000 \\
\hline Válido & 97.000 \\
\hline Perdidos & .000 \\
\hline
\end{tabular}

Fuente: Elaboración propia

Se puede observar en la tabla 6 la pertenencia del número de casos de cada uno de los conglomerados. 
En la figura 4 se muestra el resultado de las brechas en las diferentes dimensiones para evaluar las competencias docentes.

Figura 4. Dimensiones Docencia, Investigación, Tutorías y Gestión

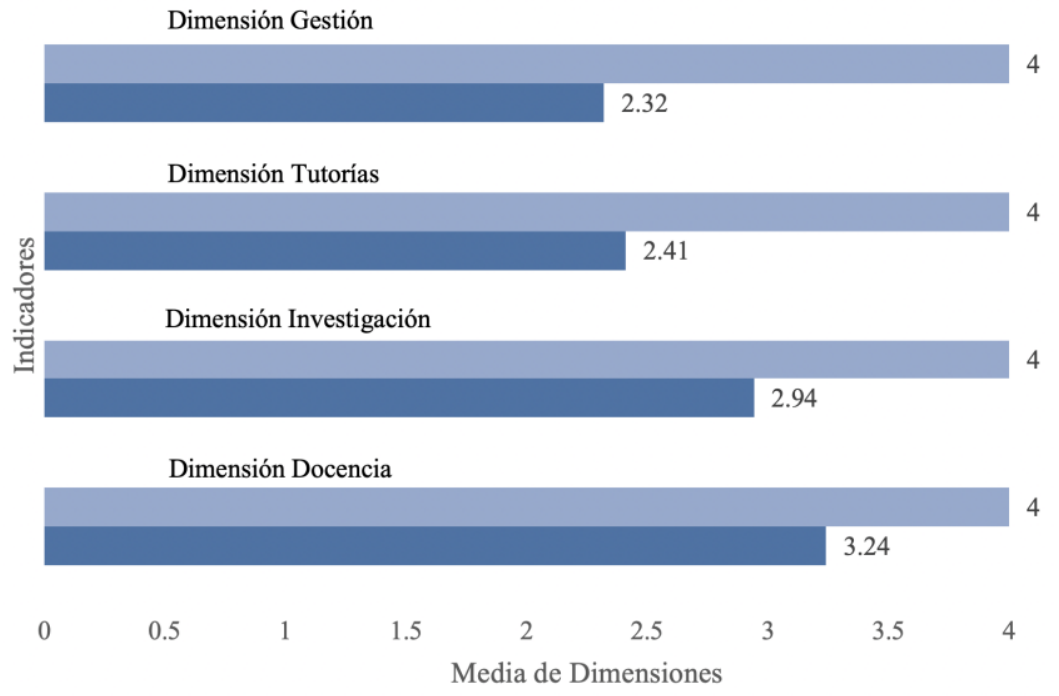

= Desarrollo Deseado $\quad$ Desarrollo Actual

Fuente: Elaboración propia

Las evidencias recolectadas en el marco teórico hacen mención de que las diferentes dimensiones no son solo un deseo, sino un requerimiento del perfil docente universitario. En el análisis de los resultados puede observarse que existen áreas de oportunidad importantes, especialmente en la investigación, tutoría y gestión. En el análisis de brechas sobre dimensiones se aprecia que la dimensión Docencia es la mejor evaluada, ya que cuenta con una calificación promedio de 3.24 de 4 , el valor máximo esperado. Además, se tienen identificadas algunas características de la dimensión Investigación, tal y como realizar y dirigir investigaciones, aunque no tanto en la publicación, trabajo colegiado y colaboración internacional. La calificación en esta dimensión es de 2.94 de 4, el valor máximo esperado, por lo que la capacitación en esta área es necesaria. En cuanto a las dimensiones Tutoría y Gestión, el área de oportunidad es mayor, ya que solamente tienen el 2.41 y 2.32, respectivamente, del esperado óptimo ya mencionado. 


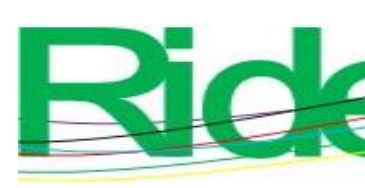

Revista Iberoamericana para la

Investigación y el Desarrollo Educativo ISSN $2007-7467$

\section{Discusión}

El modelo por competencias ha puesto al descubierto la transformación que ha experimentado el perfil del docente, un conjunto de requerimientos que probablemente antes no se utilizaban, como son los saberes de Tobón (2004), en donde se conjuntan el conocimiento, el ser y el estar, entre otros aspectos que actualmente se consideran esenciales e ineludibles para las nuevas generaciones que están demandando nuevas condiciones hacia la preparación del modelo emergente (Durán, 2016). En la carrera de Magisterio de la Escuela Universitaria de Magisterio (Escuni), en Madrid, España, se identifican cuatro competencias específicas de formación disciplinar y profesional común a todos los perfiles de maestros: la competencia cognitiva (saber), la competencia funcional (saber hacer), la competencia personal (saber estar) y la competencia ética (saber ser). Los objetivos manifiestos por la Unión Europea, así como las titulaciones de grado, están orientadas a proporcionar al estudiante universitario no solo conocimientos, sino también estrategias para seguir aprendiendo y formarse a lo largo de la vida (Megía, 2016). Es importante destacar que cualquier competencia es un saber actuar complejo, donde lo importante no son los recursos, sino la forma como la persona armoniza, forma, activa una gran variedad de recursos, intrínsecos y extrínsecos, para actuar en variedad de situaciones. Es un concepto complejo, multidimensional, por lo que identificar los rasgos más sobresalientes que la caracterizan ayuda a desentrañar su verdadera dimensión y alcance, y favorece su correcta utilización (Ruiz y Aguilar, 2017).

La evaluación docente es uno de los factores más importantes para mejorar la calidad educativa. Por lo que es necesaria una evaluación externa encaminada al desarrollo profesional en estrecha conexión con la autoevaluación habitual de los propios docentes (Marchesi y Pérez, 2018). Actualmente, para la práctica educativa, competencia es una palabra que se puede percibir más como una moda, un ideal, que como un punto de vista mediante el cual es posible situar el aprendizaje, ya que su definición deriva en confusión y ambigüedad. Por tal motivo, su instrumentación se da de manera errónea, así como su planeación, implementación y criterios de evaluación en el ámbito educativo, motivo por el que es muy significativo que el docente universitario, siendo el forjador de profesionales, tenga claridad pedagógica de ello (Rojas, Luna y Hernández, 2018).

A partir del proceso de Bolonia en Italia, se comenzó a exigir una nueva organización de la actividad académica para emprender dentro del marco social de la información y conocimiento, los desafíos procedentes de la innovación. La integración de competencias en estudios de nivel superior proporciona un elemento básico para ayudar a la formación de una sociedad en constante cambio, que reformula sus exigencias 


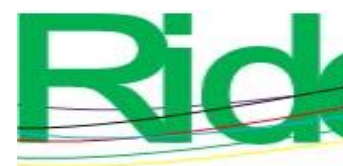

Revista Iberoamericana para la Investigación y el Desarrollo Educativo ISSN $2007-7467$

constantemente y pretende volver más profesional la formación universitaria vinculando la universidad, sociedad y el ámbito laboral. En los próximos años, el docente deberá lograr una serie de competencias que le permitan atender y solucionar situaciones problemáticas afines con una práctica creadora del cambio, a través de un desarrollo profesional, con la reconstrucción posible de la realidad y de un posicionamiento personal y profesional efectivo. Por lo tanto, es puntual el hecho de superar un tipo de visualización académica y mercantil del trabajo docente, e impulsar un modelo de enseñanzaaprendizaje basado en la reflexión y la autocrítica sobre la práctica, y así conducir al docente a un cuestionamiento de su propio ejercicio profesional. Además, debe conocer los cursos que enseña y cómo debe instruir a los alumnos, por tanto, demanda conocimientos técnicos científicos y educativos en su actividad profesional, así como capacidades de análisis y reflexión, comprensión y aplicación del proceso por medio de competencias docentes (Palacios, López y Fraile, 2019).

El análisis de la propia percepción sirve para medir la competencia a partir de alegatos y sentires del mismo sujeto, y de esta manera enfrentar las pruebas de certificación que las organizaciones buscan acreditar (Durán, Prendes y Gutiérrez, 2019). Un perfil acorde a las demandas sociales, culturales y laborales implica que todo profesional adquiera las competencias suficientes para desarrollar de un modo adecuado las ocupaciones alrededor de su puesto de trabajo. Esta necesidad queda constatada en la estructuración de los diferentes perfiles profesionales y es deber de la educación superior satisfacerla, pues se debe tener en cuenta el impacto de su quehacer laboral y la trascendencia que esto tiene en la consecución de una sociedad más equitativa y justa para todas las personas (García y Aznar, 2019).

Una integración de escenarios similares al real constituye la evaluación de las competencias docentes. Existen modelos que dan las pautas para la creación de instrumentos de evaluación del desempeño docente que den la evidencia necesaria de validez, pero no una gran fidelidad en México. La validez consiste en el nivel en que la evidencia y la teoría apoyan las formas de interpretar los resultados en una prueba para los usos presentados de una evaluación. Se requieren más herramientas de evaluación sistemática, metódica e integral, además de los modelos en evaluación que existen actualmente (Lobato, Alpuche, Trejo y Martínez, 2019). Analizar e identificar estrategias que contribuyan a potenciar programas de formación de mentores es un factor clave en la calidad educativa. Esto es un asunto difícil porque requiere la colaboración no solo del propio sistema educativo y de los planes de estudio, sino también de todos los agentes implicados e instituciones colaboradoras (Megía, 2016). En la investigación realizada de 


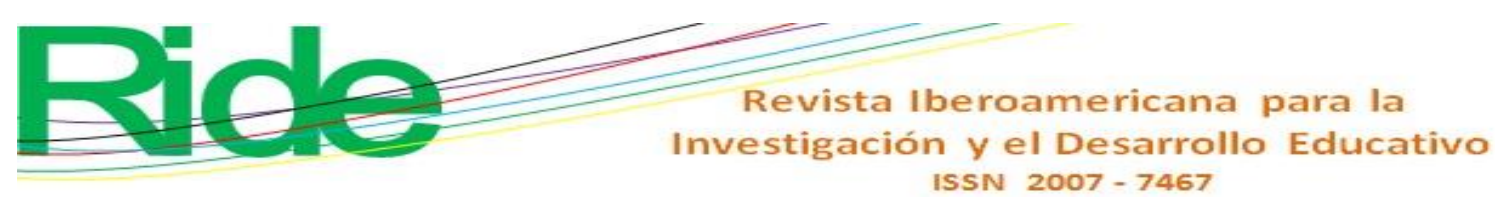

la FCA en la UACH también se tienen identificadas estrategias para lograr la potencialización de los docentes en el desarrollo de su profesión, basado en competencias necesarias para llevar a cabo su desempeño en la institución, además de cumplir con procesos de certificación.

\section{Conclusiones}

En la FCA de la UACH se está trabajando en la formación constante de docentes de manera sistemática. La definición del perfil de competencias docentes que se ha evaluado contribuirá notablemente en fortalecer la plantilla docente, al integrar innovaciones en un programa de formación que de este estudio se desprenda, ya que esta investigación repercutirá también como un instrumento de diagnóstico que apunta a generar mejores prácticas educativas. La educación de nivel superior debe evolucionar para ser de mejor calidad; no solamente es necesaria la innovación, sino también reforzar las áreas que debieran estar consolidadas. Es un compromiso con los alumnos y la comunidad en general, ya que los futuros profesionistas deben salir preparados para el desempeño de sus actividades profesionales, pero también deben ser ciudadanos responsables y comprometidos con la sociedad en que viven. De la misma forma, también es un compromiso de la institución educativa con sus docentes.

Con base en los datos presentados, es necesaria la elaboración de planes de formación dirigidos específicamente a las distintas etapas de la vida profesional. Son necesarios planes formativos dirigidos a los nuevos docentes y a aquellos que son más experimentados, planes de seguimiento e innovación a la experiencia ya adquirida y también en las diferentes necesidades de capacitación y desarrollo que son requeridas en las dimensiones en que se compone el quehacer docente y su desempeño. Aquí se detectaron necesidades específicas en las dimensiones de Docencia, que es la mejor evaluada, seguida de la dimensión de Investigación; las más rezagadas, la de Tutoría y Gestión, donde la oportunidad de desarrollarse es más amplia y urgente. 


\section{Recomendaciones}

Después de la realización de un análisis de la evaluación de competencias docentes, existe un área de oportunidad sobre la capacitación y desarrollo de los profesores. Se destaca una necesidad de involucramiento a la planta docente en el plano de investigación, así como combatir un importante rezago en la tutoría y gestión, ya que presenta una insuficiencia que debe ser atendida. También se recomienda que dichas capacitaciones se realicen involucrando a docentes de todas las edades y formas de situación laboral, donde convivan y compartan todas las experiencias e innovaciones entre compañeros docentes, para elevar la calidad educativa de la institución y en beneficio de todos.

\section{Limitaciones del estudio}

Se tiene la limitante del cambio de modelo educativo que se implementó en agosto de 2019, y la información aquí utilizada corresponde al modelo anterior. Sin embargo, cabe señalar que, aunque existan algunos cambios en el modelo educativo en la FCA de la UACH, este seguirá incluyendo las competencias.

\section{Futuras líneas de investigación}

Evaluar las competencias docentes periódicamente para generar estrategias y acciones para capacitar a los profesores en las áreas de oportunidad. 


\section{Referencias}

Ashwin, P. (2006). Changing Higher Education. The Development of Learning \& Teaching. London, England: Routledge.

Brunner, J. J. (1994). Educación superior en América Latina: una agenda de problemas, políticas y debates en el umbral del año 2000. Buenos Aires, Argentina: Consejo Latinoamericano de Ciencias Sociales.

Carrera, C. y Marín, R. (2011). Modelo pedagógico para el desarrollo de competencias en educación superior. Revista Electrónica Actualidades Investigativas en Educación, 11(1), 1-32.

Carrera, F., Vaquero, E. y Balsells, M. (2011). Instrumento de evaluación de competencias digitales para adolescentes en riesgo social. Edutec. Revista Electrónica de Tecnología Educativa, (35), 1-25. Recuperado de https://www.edutec.es/revista/index.php/edutec-e/article/view/410.

Comisión Nacional de Acreditación de Chile (2016). Criterios de evaluación para la acreditación de carreras profesionales, carreras profesionales con licenciatura y programas de licenciatura. Recuperado de www.cnachile.cl /Paginas/Acreditacion-Pregrado.aspx.

Cuadra, D, Castro, P. J. y Juliá, M. T. (2018). Tres saberes en la formación profesional por competencias: integración de teorías subjetivas, profesionales y científicas. Formación Universitaria, 11(5), 19-30. Recuperado de https://dx.doi.org/10.4067/S0718-50062018000500019.

Dios, I., Calmaestra, J. y Rodríguez, A. (2018). Validación de la escala de competencias docentes organizacionales y didácticas para educadores. Revista Mexicana de Investigación Educativa, 23(76), 281-302.

Durán, A. A. (2016). Formación en competencias del docente universitario. Educere, 20(67), 529-538.

Durán, M., Prendes, M. y Gutiérrez, I. (2019). Certificación de la competencia digital docente: propuesta para el profesorado universitario. RIED. Revista Iberoamericana de Educación a Distancia, 22(1), 187-205.

Esteve, F., Castañeda, L. y Adell, J. (2018). Un modelo holístico de competencia docente para el mundo digital. Revista Interuniversitaria de Formación del Profesorado, 32(1), 105-116. Recuperado de https://dialnet.unirioja.es/servlet/articulo?codigo $=6441415$. 
Fernández, L., Díaz, L. y Leyva, E. (2016). Competencias genéricas en docentes del nivel superior en el Estado de Guerrero. RIDE Revista Iberoamericana para la Investigación y el Desarrollo Educativo, 6(12).

Fondo, M. (2019). Seis competencias docentes clave para el siglo XXI. marcoELE. Revista de Didáctica Español Lengua Extranjera, (29), 1-14.

García, Z. y Aznar, I. (2019). El desarrollo de competencias investigativas, una alternativa para formar profesionales en pedagogía infantil como personal docente investigador. Revista Electrónica Educare, 23(1),1-22.

García, C. M. y Vaillant, D. (2010). Desarrollo profesional docente: ¿cómo se aprende a enseñar? (Vol. 115). Narcea Ediciones.

Guzmán, I., Marín R. e Inciarte, A. (2014). Innovar para transformar la docencia universitaria. Un modelo para la formación de competencias. Maracaibo, Venezuela: Universidad del Zulia.

Hernández, H. A., y Pascual, A. E. (2018). Validación de un instrumento de investigación para el diseño de una metodología de autoevaluación del sistema de gestión ambiental. Revista de Investigación Agraria y Ambiental, 9(1), 157-164. Recuperado de https://doi.org/10.22490/21456453.2186.

Inche, J. y Chung, A. (2012). Estudio prospectivo para la enseñanza superior virtual al 2030. Industrial Data, 15(1), 120-126. Recuperado de https://doi.org/10.15381/idata.v15i1.6260.

Lobato, V. M., Alpuche, H. A., Trejo, M. J. y Martínez, G. A. (2019). Desarrollo de evidencias de validez de una prueba de desempeño sobre competencias docentes: EDOE. Investigación en Educación Médica, 8(29),45-57.

Marchesi, Á. y Pérez, E. M. (2018). Modelo de evaluación para el desarrollo profesional de los docentes. Madrid, España: Fundación SM.

Marín, R. (2003). El modelo educativo de la UACH: elementos para su construcción. México: UACH/Dirección académica.

Marín, R., Guzmán, I., Márquez, A. y Peña, M. (2013). La evaluación de competencias docentes en el modelo DECA: anclajes teóricos. Formación Universitaria, 6(6), 41-54.

Mas, Ò. (2011). El Profesor Universitario: sus competencias y formación. Profesorado. Revista de Currículum y Formación de Profesorado, 15(3),195-211. ISSN: 1138414X. Disponible en: https://www.redalyc.org/articulo.oa?id=56722230013

Mas, Ò. y Olmos, P. (2016). El profesor universitario en el espacio europeo de educación superior. La autopercepción de sus competencias docentes actuales y 


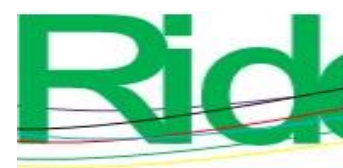

Revista Iberoamericana para la Investigación y el Desarrollo Educativo ISSN 2007 - 7467

orientaciones para su formación pedagógica. Revista Mexicana de Investigación Educativa, 21(69), 437-470.

Megía, C. (2016). Competencias del profesor mentor del aprendiz de maestro. Una propuesta de formación. (Tesis de doctorado). Universidad Nacional de Educación a Distancia.

Montenegro, H. y Fuentealba, R. (2010). El formador de futuros profesionales: una nueva forma de comprender la docencia en la educación superior universitaria. Calidad en la Educación, (32), 253-267.

Palacios, A., López, V. y Fraile, A. (2019). Cuestionario de percepción de competencias docentes de Educación Física. Revista Internacional de Medicina y Ciencias de la Actividad Física y del Deporte, 19(75).

Valles, H., Parra, H., Guzmán, I., Marín, R., Delgado, C., Marín-Trejo, R., Avitia, Y., Arias, R., Piñón, L., Gutiérrez, M.C., Sapién, L., Soto, M. y Franco, P. (2017). Competencias académicas del profesor universitario. Chihuahua, México: Universidad Autónoma de Chihuahua.

Rojas, A., Luna, J. y Hernández, I. (2018). Competencias docentes transversales para el quehacer de profesores de pregrado en Psicología. Educación y Educadores, 21(3), 461-481. Recuperado de https://educacionyeducadores.unisabana.edu.co/index.php/eye/article/view/8506.

Rojas, I. (2000). La educación basada en normas de competencia (EBNC) como un nuevo modelo de formación profesional en México. En Valle, M. (coord. $\left.{ }^{a}\right)$, Formación en competencias y certificación profesional (pp. 45-75). Ciudad de México, México: Universidad Nacional Autónoma de México.

Ruiz, M. y Aguilar, R. (2017). Competencias del profesor universitario: elaboración y validación de un cuestionario de autoevaluación. Revista Iberoamericana de Educación Superior, 8(21), 37-65.

Tobón, S. (2004). Formación basada en competencia: Pensamiento complejo, diseño curricular y didáctica (1. ${ }^{\mathrm{a}}$ ed.). Bogotá, Colombia: Ecoe Ediciones.

Toro, I., Saldarriaga, J., León, M., Martínez, J. y Arias, O. (2015). Competencias docentes para la enseñanza de la metodología de la investigación y la evaluación de trabajos de grado y tesis doctorales en administración. El Ágora USB, 15(1), 137-151. Recuperado de https://doi.org/10.21500/16578031.7. 


\begin{tabular}{|c|c|}
\hline Rol de Contribución Autor (es) & Autor (es) \\
\hline Conceptualización & $\begin{array}{l}\text { Alma Lilia Sapién Aguilar «principal» } \\
\text { Laura Cristina Piñón Howlet «igual» } \\
\text { Silvia Patricia Aguirre Espino «igual» } \\
\text { Luis Antonio Molina Corral «que apoya» }\end{array}$ \\
\hline Metodología & Laura Cristina Piñón Howlet \\
\hline Análisis Formal & $\begin{array}{l}\text { Alma Lilia Sapién Aguilar «igual» } \\
\text { Silvia Patricia Aguirre Espino «igual» }\end{array}$ \\
\hline Investigación & Laura Cristina Piñón Howlet \\
\hline Recursos & Alma Lilia Sapién Aguilar \\
\hline Curación de datos & Silvia Patricia Aguirre Espino \\
\hline $\begin{array}{l}\text { Escritura - Preparación del } \\
\text { borrador original }\end{array}$ & $\begin{array}{l}\text { Alma Lilia Sapién Aguilar «igual» } \\
\text { Laura Cristina Piñón Howlet «igual» } \\
\text { Silvia Patricia Aguirre Espino «igual» }\end{array}$ \\
\hline Escritura - Revisión y edición & $\begin{array}{l}\text { Alma Lilia Sapién Aguilar «igual» } \\
\text { Laura Cristina Piñón Howlet «igual» } \\
\text { Luis Antonio Molina Corral «que apoya» }\end{array}$ \\
\hline Visualización & $\begin{array}{l}\text { Alma Lilia Sapién Aguilar «igual» } \\
\text { Laura Cristina Piñón Howlet «igual» } \\
\text { Silvia Patricia Aguirre Espino «igual» }\end{array}$ \\
\hline Supervisión & $\begin{array}{l}\text { Alma Lilia Sapién Aguilar «igual» } \\
\text { Laura Cristina Piñón Howlet «igual» } \\
\text { Luis Antonio Molina Corral «que apoya» }\end{array}$ \\
\hline Administración de Proyectos & Alma Lilia Sapién Aguilar \\
\hline Adquisición de fondos & $\begin{array}{l}\text { Alma Lilia Sapién Aguilar «igual» } \\
\text { Laura Cristina Piñón Howlet «igual» } \\
\text { Luis Antonio Molina Corral «igual» }\end{array}$ \\
\hline
\end{tabular}

